\title{
MORPHOLOGICAL EVALUATION OF GILT OVARIES IN RELATION TO THE QUALITY OF RECOVERED OOCYTES
}

\author{
D. ABADJIEVA, TS. GOSPODINOVA, R. STEFANOV \& E. KISTANOVA
}

Department of Embryobiotechnology of Animals, Institute of Biology and Immunology of Reproduction, Bulgarian Academy of Sciences, Sofia, Bulgaria

\begin{abstract}
Summary
Abadjieva, D., Ts. Gospodinova, R. Stefanov \& E. Kistanova, 2015. Morphological evaluation of gilt ovaries in relation to the quality of recovered oocytes. Bulg. J. Vet. Med., 18, No 3, 209-215.

The aim of the current study was to analyse the relationship between morphology of gilt's ovary and the quantity and quality of recovered oocytes suitable for in vitro procedures. Forty ovaries of healthy 7-8 months old gilts from a local slaughterhouse divided into corpora lutea bearing (CL) and noncorpora lutea bearing (non-CL) ovaries were evaluated. Morphometric analysis (weight, length and width) of the ovaries was performed. Also, visual estimation of the number and size of follicles and presence of corpora lutea in ovaries was done. The developmental competence of recovered oocytes was evaluated by BCB test. It was found that the values of morphometric parameters of CL ovaries were higher compared to non-CL ovaries $(\mathrm{P}<0.05)$. Non-CL ovaries tended to bear a higher number of large and total follicles than CL, but the difference was not significant. More compact cumulus oocytes complexes (COCs) and oocytes with corona radiata (POCs) were recovered from CL than from non-CL ovaries. The BCB test showed that both types of ovaries provided more than $55 \% \mathrm{BCB}-$ positive oocytes. In conclusion, both $\mathrm{CL}$ as well as non-CL ovaries from gilts aged 7-8 months could be used for recovering the developmental competent oocytes for in vitro procedures.
\end{abstract}

Key words: corpora lutea (CL), gilt ovary, oocytes

\section{INTRODUCTION}

The in vitro production of mammalian embryos has a broad range of applications: from production of cloned animals and genetic engineering to treatment of infertility by assisted reproductive technologies. Recently, there has been an increasing interest in producing large quantities of pig embryos through in vitro maturation (IVM) in vitro fertilisation (IVF) techniques because of the physio- logical similarities of pig oocytes to those of humans, which makes these animals a valuable experimental model for fundamental as well as for biomedical research (Prather et al., 2008).

The pre-requisite for high percentage of successful in vitro procedures (IVP) is the availability of a sufficient number of oocytes. In this case, the ovaries from slaughtered gilts are not only suitable, but 
the cheapest and the most abundant source of oocytes.

During the last decades an extensive research on IVM/ IVF in pigs resulting in zygotes producing has been done (Gil et al., 2013). However, the information about the evaluation of ovaries as a primary source for the efficient collection and grading of oocytes is limited.

The investigations of Marchal et al. (2001) and Bagg et al. (2004) had shown that there was a difference between oocytes from the ovaries of gilts and sows: despite the similar fertilisation rate the oocytes from gilts rarely develop into blastocyst. In a later study, Bagg et al. (2007) reported that only oocytes from big follicles of gilt ovaries reached the blastocyst stage. This means that the optimal rates of embryo in vitro production can be attained by selecting high quality follicles (Wright, 2012).

Usually the gilts are slaughtered after reaching 100-120 $\mathrm{kg}$ body weight at the age of 7-8 months. Although the approximately similar age, the animals are not in a similar stage of sexual development, hence the different physiological and morphological state of ovaries. The aim of the current study was to analyse the relationship between of the gilt ovary morphology and the quantity and quality of recovered oocytes suitable for in vitro procedures.

\section{MATERIALS AND METHODS}

\section{Collection of ovaries}

Forty ovaries from clinically healthy gilts aged 7-8 months weighing approximately $120 \mathrm{~kg}$, with unknown reproductive history were obtained from a slaughterhouse during May and June 2014. Pairs of ovaries from a pig were collected in a thermos at $37{ }^{\circ} \mathrm{C}$ containing $0.9 \%$ physiologi- cal saline and transported to the laboratory in the Institute of Biology and Immunology of Reproduction, Bulgarian Academy of Sciences within 1-2 hours after slaughter.

Morphological and morphometric evaluation

Upon arrival at the laboratory the ovaries were washed three times in phosphate buffered saline prepared according to Gordon (1994). By visual estimation ovaries were divided in two groups depending on the presence or absence of corpora lutea (CL): CL bearing and non-CL bearing (Fig. 1).

The morphometric parameters of the ovary - weight, length and width were measured. The length of each ovary was determined as the maximum distance from pole to pole along an axis parallel to the ovarian mesenteric attachment. The width was determined as the furthest distance along an axis vertical to the longitudinal axis. The number and size of follicles per each ovary were determined before puncture. The follicles were characterised on the basis of their diameter as small (2-6 $\mathrm{mm})$, and large ( $\geq 6 \mathrm{~mm})$.

\section{Oocyte recovery and classification}

All visible follicles from the ovarian surface were aspirated with an 18-gauge sterile needle fixed on a syringe. The flushing medium was phosphate buffered saline (PBS) supplemented with $0.4 \%$ lyophilised bovine serum albumin (BSA; Sigma Chemical Co., St. Louis, MO, USA). The oocytes were collected under stereomicroscope (Nikon SMZ-10, Japan), and were washed three times in the flushing media. The recovered oocytes (Fig. 2) were classified according to their morphological shape into COCs, oocytes with homogenous evenly granulated cytoplasm pos- 
sessing at least three layers of cumulus cells, oocytes partially denuded of cumulus cells and/or with irregular shrunken cytoplasm (POCs), oocytes completely denuded of cumulus cells and/or with ir-
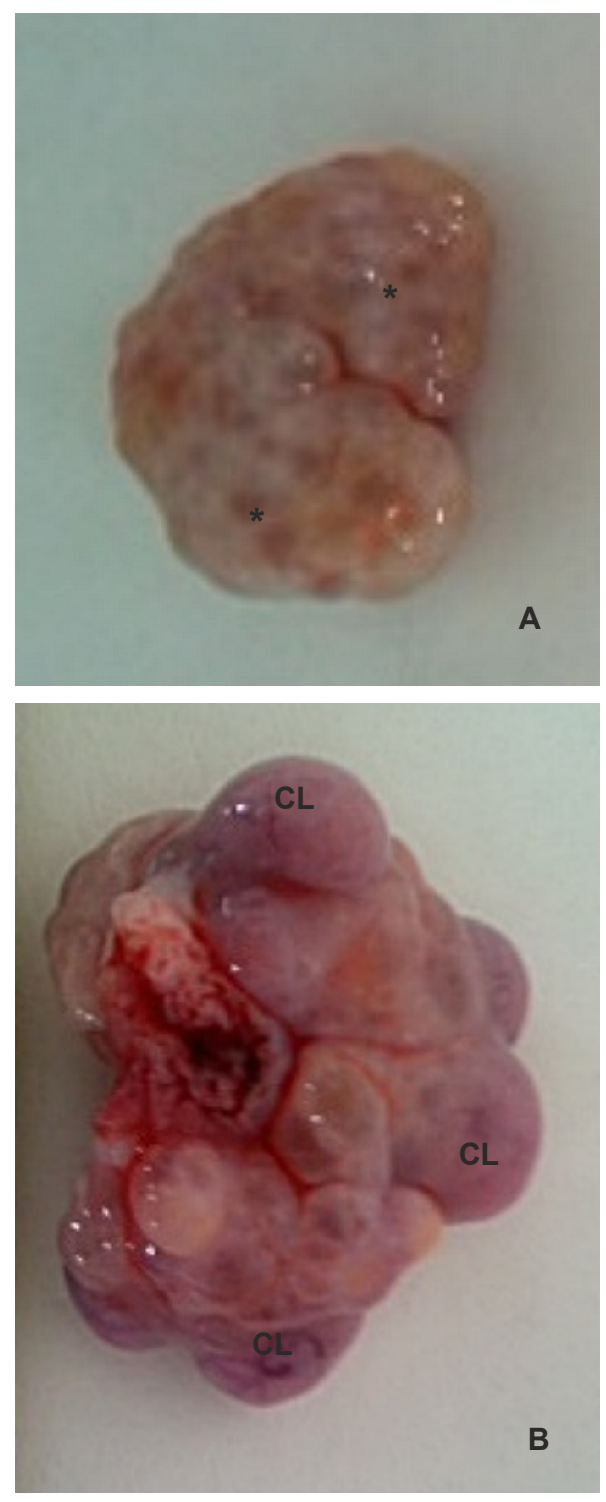

Fig. 1. Gilt ovary morphology: A. Non-corpora lutea bearing ovaries with small follicle $(*)$; B. Corpora lutea $(\mathrm{CL})$ bearing ovaries. irregular shrunken cytoplasm (DO), (Chauhan et al., 1998).

Evaluation of COCs by the brilliant cresyl blue staining $(B C B)$ test

The brilliant cresyl blue (BCB) staining is method which determines the intracellular activity of glucose-6-phosphate dehydrogenase, a pentose phosphate pathway enzyme that gradually decreases its activity as oocytes reach their growth phase. Fifteen COCs from each group of ovaries were washed twice in PBS and tested in BCB solution (Merck KGaA, Darmstadt, Germany) in concentration $13 \mu \mathrm{M}$ with subsequent incubation for $90 \mathrm{~min}$ at $39^{\circ} \mathrm{C}$ in a humidified $5 \% \mathrm{CO}_{2}$ atmosphere $(\mathrm{El}$ Shourbagy et al., 2006). After incubation, the oocytes were transferred to PBS supplemented with $0.4 \%$ BSA and washed twice. The washed oocytes were examined under stereomicroscope and classified into two groups: those with blue-stained ooplasm were evaluated as $\mathrm{BCB}$ positive $(\mathrm{BCB}+)$, and those with a colourless ooplasm - as BCB negative (BCB-).

\section{Statistical analysis}

Data are presented as mean \pm standard deviation (SD). The $P$ value less than 0.05 was considered as significant. All statistical analyses were performed using the statistical package SPSS for Windows v. 16.0.1.

\section{RESULTS}

Out of the investigated 40 ovaries $\mathrm{CL}$ presence was exhibited in $22(55.0 \%)$ ovaries, the other 18 were non-CL bearing. Data presented in Table 1 showed the mean weight, length and width of the evaluated ovaries. The numeric values of morphometric parameters of CL bearing 

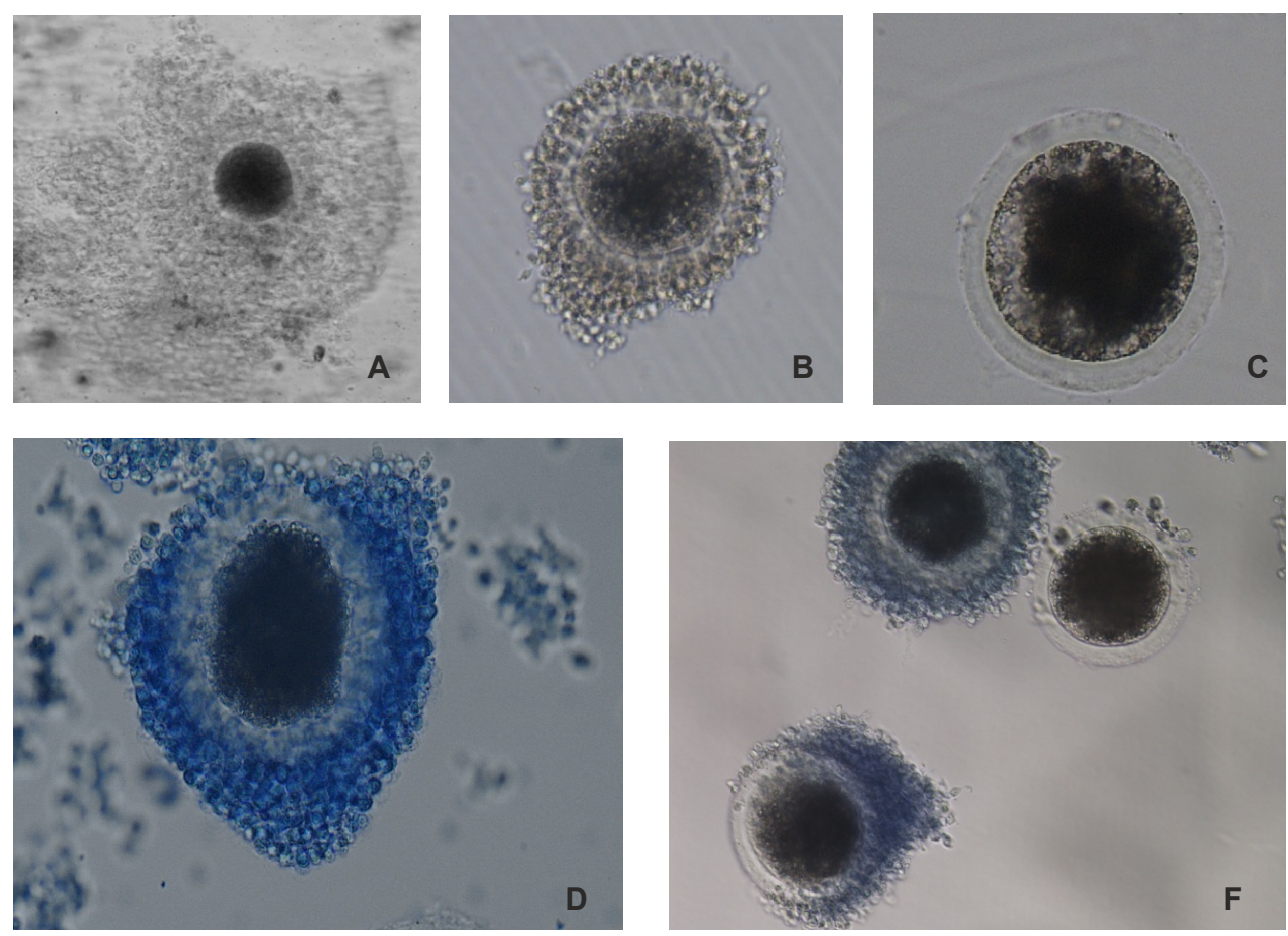

Fig. 2. The collected gilt oocytes: A. compact cumulus oocytes complexes, $\mathrm{COCs}(\times 10)$; B. oocytes partially denuded of cumulus cells $(\times 20)$; C. denuded oocytes $(\times 20)$; D. BCB test on COCs from non-CL ovaries $(\times 20)$; E. BCB test on COCs from CL ovaries $(\times 20)$.

Table 1. Morphometric data of gilt ovaries. Data are presented as mean $\pm \mathrm{SD}$

\begin{tabular}{llc}
\hline Parameters & & P value \\
\hline Weight of the ovary $(\mathrm{g})$ & & \\
CL bearing ovaries $(\mathrm{n}=22)$ & $3.62 \pm 0.45$ & 0.0002 \\
non-CL bearing ovaries $(\mathrm{n}=18)$ & & \\
Length of the ovary $(\mathrm{cm})$ & $2.85 \pm 0.14$ & 0.0001 \\
CL bearing ovaries $(\mathrm{n}=22)$ & $2.53 \pm 0.08$ & \\
non-CL bearing ovaries $(\mathrm{n}=18)$ & & \\
Width of the ovary $(\mathrm{cm})$ & $1.78 \pm 0.10$ & 0.0001 \\
CL bearing ovaries $(\mathrm{n}=22)$ & $1.34 \pm 0.13$ & \\
non-CL bearing ovaries $(\mathrm{n}=18)$ & & \\
\hline
\end{tabular}

ovaries were significantly higher than parameters of non-CL ovaries $(\mathrm{P}<0.001)$.

Non-CL bearing ovaries tended to bear a higher number of total and large follicles than those of CL bearing ovaries (Table
2). The number of small follicles per ovary was similar in both groups of ovaries.

CL bearing ovaries provided a higher percentage of COCs and oocytes partially 
denuded of cumulus cells $(84 \%)$ in comparison with non-CL ovaries $(80 \%)$. From the non-CL ovaries more fully denuded oocytes $(20 \%$ against $16 \%)$ were recovered (Table 3).

BCB staining test demonstrated that the both groups of ovaries produced high percent of $\mathrm{BCB}+$ oocytes - more than $55 \%$. At the same time it should be noticed that non-CL ovaries provided a significantly higher number of $\mathrm{BCB}+$ oocytes than CL ovaries (78\% against $60 \%$, $\mathrm{P}=0.008$ ) (Fig. 2D, E, F).

\section{DISCUSSION}

The present study was focused on the evaluation of gilt ovary parameters related to the quality of porcine oocytes. There are few studies focused on the investigation of differences in oocytes quality obtained from ovaries of sows and gilts (Marshal et al., 2001; Bagg et al., 2004,
2007; Boland et al., 2011). The research had shown that the competence of recovered oocytes depended on the morphological and physiological state of ovaries.

Pawlak et al. (2011) demonstrated that the puberty of slaughtered gilts affected the quality of oocytes. The presence of corpora lutea in the ovary is a sign of cycling gilts that have reached the puberty. In our study the ovaries were evaluated on the base of presence or absence of corpora lutea. It was revealed that the morphometric parameters (weight, length and width) of CL bearing were significantly higher than those of non-CL bearing ovaries. The reason may be a major portion of lutein cells in the CL bearing ovaries (Kumar et al., 1997). Guimarães et al. (2004) presented results related to the ovary weight (4.40-4.70 g) similar to ours $(3.73-4.62$ g.). The measured parameters of ovarian length and width were comparable to those reported by Bagg et al. (2004) in pre-pubertal gilts. The ovary

Table 2. Follicular development in corpora lutea (CL) and non-CL bearing gilt ovaries. Data are presented as mean $\pm \mathrm{SD}$

\begin{tabular}{lccc}
\hline Parameters & $\begin{array}{c}\text { CL bearing ovary } \\
(\mathrm{n}=22)\end{array}$ & $\begin{array}{c}\text { Non-CL bearing ovary } \\
(\mathrm{n}=18)\end{array}$ & P value \\
\hline Total number of follicles & $15.39 \pm 0.61$ & $16.27 \pm 0.74$ & 0.0095 \\
Small follicles $(2-6 \mathrm{~mm})$ & $10.11 \pm 0.46$ & $10.15 \pm 0.70$ & 0.8816 \\
Large follicles $(\geq 6 \mathrm{~mm})$ & $4.48 \pm 0.18$ & $4.86 \pm 0.33$ & 0.0050 \\
\hline
\end{tabular}

Table 3. Distribution of the oocytes recovered from corpora lutea (CL) and non-CL ovaries according to their morphological shape. Data are presented as mean $\pm \mathrm{SD}$

\begin{tabular}{lccc}
\hline Parameters & $\begin{array}{c}\text { CL bearing ovary } \\
(\mathrm{n}=22)\end{array}$ & $\begin{array}{c}\text { Non-CL bearing ovary } \\
(\mathrm{n}=18)\end{array}$ & P value \\
\hline COCs & $20(42 \%)$ & $40(40 \%)$ & 0.89 \\
POCs & $20(42 \%)$ & $40(40 \%)$ & 0.37 \\
DO & $8(16 \%)$ & $20(20 \%)$ & 0.07 \\
\hline Total & $48(100 \%)$ & $100(100 \%)$ & \\
\hline
\end{tabular}

COCs: compact cumulus oocytes complexes; POCs: oocytes partially denuded of cumulus cells; DO: oocytes completely denuded of cumulus cells. 
without CL exhibited better follicular activity, had significantly higher total number of follicles and large follicles than that CL bearing ovary. This alteration was attributed to differences in glucose metabolism and active proteins, responsible for ovarian development (Paczkowski \& Krisher, 2010).

The current study demonstrated that the presence of CL in the ovary affected the developmental quality of oocytes. Despite the higher percent of quality COCs recovered from $\mathrm{CL}$ bearing ovaries, they provided more $\mathrm{BCB}-$ than $\mathrm{BCB}+$ oocytes. $\mathrm{BCB}$ - could be a marker for the oocytes' potency to grow, whereas $\mathrm{BCB}+$ is a proof that oocytes have completed their growth. $\mathrm{BCB}-$ oocytes, unlike $\mathrm{BCB}+$, have a higher activity of G6PDH that is still necessary to complete the unfinished chromatin remodelling process. Investigations showed that porcine $\mathrm{BCB}+$ oocytes often reach the MII stage and possess higher fertilisation rate (Roca et al., 1998; Pawlak et al., 2011). These data confirmed the reliability of the BCB test as an additional means for estimation of the porcine oocytes quality.

In summary, the ovaries without CL (non-cycling/pre-pubertal gilts) provide a large number of follicles as well as good quality of COCs similar to those obtained from ovaries bearing CL (cycling/pubertal gilts). In both groups of ovaries the prevalence of $\mathrm{BCB}+$ oocytes was omore than $55 \%$ indicating that ovaries from prepubertal gilts could also be used to collect quality COCs for in vitro procedures.

\section{REFERENCES}

Bagg, M. A., R. Vassena, E. Papasso-Brambilla, C. G. Grupen, D. T. Armstrong \& F. Gandolfi, 2004. Changes in ovarian, follicular, and oocyte morphology immediately after the onset of puberty are not ac- companied by an increase in oocyte developmental competence in the pig. Theriogenology, 62, 1003-1011.

Bagg, M. A., M. B. Nottle, D. T. Armstrong \& C. G. Grupen, 2007. Relationship between follicle size and oocyte developmental competence in prepubertal and adult pigs. Reproduction Fertility Development, 19, 797-803.

Boland, M. P., P. Lonergan \& D. O'Callaghan, 2001. Effect of nutrition on endocrine parameters, ovarian physiology and oocyte and embryo development. Theriogenology, 55, 1323-1340.

Chauhan, M. S., S. K. Singla, P. Palta, R. S. Manik \& M. L. Madan, 1998. In vitro maturation and fertilization, and subsequent development of buffalo (Bubalus bubalis) embryos: Effects of oocyte quality and type of serum. Reproduction Fertility Development, 10, 173-177.

El Shourbagy, S. H., E. C. Spikings, M. Freitas \& J. C. St John, 2006. Mitochondria directly influence fertilisation outcome in the pig. Reproduction, 131, $233-$ 245.

Gil, M. A., J. Gomis, M. A. Angel, J. SanchezOsorio, C. Maside, C. Cuello, I. Parrilla, J. Roca, J. M. Vazquez \& E. A. Martinez, 2013. The in vitro and in vivo developmental capacity of selected porcine monospermic zygotes. Theriogenology, 79, 392-398.

Gordon, I., 1994. Laboratory Production of Cattle Embryos. CAB International, University Press, Cambridge, p. 26-27.

Guimarães, G. C., M. R. F. Machado \& A. L. Q. Santos, 2004. Variáveis morfológicas dos órgãos genitais femininos de suínos da raca Landrace. Bioscience Journal, 20, 131-136.

Kumar, A., V. S. Solanki, S. K. Jindal, V. N. Tripathi \& G. C. Jain, 1997. Oocyte retrieval and histological studies of follicular population in buffalo ovaries. Animal Reproduction Science, 47, 189-195.

Marchal, R., J. M. Feugang, C. Perreau, E. Venturi, M. Terqui \& P. Mermillod, 2001. Meiotic and developmental competence of 
prepubertal and adult swine oocytes. Theriogenology, 56, 17-29.

Paczkowski, M. \& R. Krisher, 2010. Aberrant protein expression is associated with decreased developmental potential in porcine cumulus-oocyte complexes. Molecular Reproduction and Development, 77, 51-58.

Pawlak, P., N. Renska, E. Pers-Kamczyc, E. Warzych \& D. Lechniak, 2011. The quality of porcine oocytes is affected by sexual maturity of the donor gilt. Reproductive biology, 11, 1-18.

Prather, R. S., M. D. Shen \& Y. F. Dai, 2008. Genetically modified pigs for medicine and agriculture. Biotechnology and $\mathrm{Ge}$ netic Engineering Reviews, 25, 245-265.

Roca, J., E. Martinez, J. M. Vazquez \& X. Lucas, 1998. Selection of immature pig oocytes for homologous in vitro penetration assays with the brilliant cresyl blue test. Reproduction Fertility Development, 10, 479-485.
Wright, E. C., 2012. MicroRNA expression and function during porcine oocyte maturation and early embryonic development. Graduate Theses and Dissertations. Paper 12522, p. 3-24.

Paper received 13.10.2014; accepted for publication 22.01.2015

\section{Correspondence:}

Dessislava Abadjieva

Department of Embryobiotechnology of Animals, Institute of Biology and Immunology of Reproduction,

Bulgarian Academy of Sciences, 73 Tsarigradsko shose blvd,

1113 Sofia, Bulgaria e-mail: dessi_1@abv.bg 